\title{
Research on the Anaerobic Capacity of Vertical Jump and Straight Line Sprint of Basketball players in the Test
}

\author{
LI HUA ${ }^{1, a}$, LIU JING ${ }^{1, b}$ \\ ${ }^{1}$ Yunnan Yuxi normal university Sports college, 653100, China \\ a lihua913@yxnu.net, ${ }^{b}$ liujing@yxnu.net
}

Keywords: Basketball player, Vertical jump, straight line sprint, anaerobic capacity, T / C test, IAT test, Quantity statistical test

\begin{abstract}
Training of basketball athletes is different with sport training of other athletes. Basketball game requires athletes have good bounce, explosiveness, endurance after the game. Therefore, athletes should increase the proportion of anaerobic training project in the peacetime of training. Serum testosterone $\mathrm{T}$ and serum cortical $\mathrm{C}$ value will change in the process of anaerobic exercise. The individual anaerobic queue IAT value will change too. The changes of the value are related with the strength of the sport and the training time. This paper analyzes the vertical jump and the linear sprint anaerobic capacity of basketball athletes in training process.
\end{abstract}

\section{Introduction}

In modern sports, in addition to power and speed of the competition, there is also the comparison of the endurance, and athletes' anaerobic ability is one of the key elements to win the competition $[1,2]$. In basketball games, a successful performance mainly dependent on several fitness adaptability part (for example, speed, agility and vertical leap). The movement is in essence anaerobic properties, and these components must be repeated, with the lowest reduce competition for the performance. Basketball players can get better endurance and explosive through anaerobic capacity training which is the basis of the cultivation of outstanding basketball players. $\mathrm{T}$ and $\mathrm{C}$ are keep balance in the static state of the body [3]. In the process of anaerobic exercise, $T$ value is high. IAT is one of the major biochemical indicators of the guidance of aerobic training project. It can measure anaerobic endurance of a variety of sports of T / C and IAT values of basketball players' blood during training. The study of IAT is limited in the study of interval time of IAT. There are no studies of the combination of aphasic changes of $\mathrm{T} / \mathrm{C}$ values and the interval time dynamic changes of IAT. In this study, the paper finds that in the training of anaerobic endurance, $\mathrm{T} / \mathrm{C}$ values of outstanding basketball player will change. The relatively short time, IAT power will increase or decrease. In the training of anaerobic endurance, this paper detects the blood T, blood C and IAT of outstanding basketball players and analyzes the dynamic variation of $\mathrm{T} / \mathrm{C}$ values and the IAT. It aims to provide a theoretical basis for reasonable arrangements of anaerobic endurance training of outstanding basketball players $[4,5]$.

\section{Anaerobic training physiology model of vertical jump and straight line sprint}

Anaerobic training process of basketball player involves a lot of physiological knowledge of kinesiology. The most important is the change of energy. In the process of aerobic exercise, glucose transfers into pyruvate and continue transfers into CoA. Anaerobic stage will change into lactic acid. In 
the process of anaerobic training, muscles will change with the irritation of time. In Initial training stage, the amount of lactic acid will increase gradually. However, with the increase of training intensity, lactic gets to balance gradually and has no increase.

Vertical leap is measured with backward motion (CMJ) vertical leap of the maximum. All testers stand in the rubber contact platform $(120 \mathrm{~cm} * 380 \mathrm{~cm})$, and a cable is connected to a digital timer, to record jumping time in the testing. The timer for the test of the feet and then touching the ground namely stop recording. Jumping time is used to calculate the centre of the human body height change. We will record the highest CMJ height value. Anaerobic ability to jump over the experiment (APJT) also through the 15 seconds of vertical leap will make the measurement. Anaerobic ability will be calculated through the general jump time and the number of jumping movement. Linear sprint is a common site test for basketball players, which can be used to measure individual anaerobic ability. Linear sprint is conducted in the normal basketball court for sprint. Firstly, the testers stand on the base, with the fastest speed in free throws to nearby line $(5.8 \mathrm{~m})$, a half line $(14.3 \mathrm{~m})$, far from $(22.9 \mathrm{~m})$, and far baseline (28.7 meters) of separation of the cone position. They will reach the cone position, namely the sprint to return to the baseline, and reach the next line. Sprint line in three times (T1, T2 and T3), and in every time the sprint have 2 minutes to rest. Fatigue index (FILD) is produced by the slowest and the fastest record in those three times. The update speed of oxygen of vertical jump is slower than the straight jump. The update speed of oxygen reduces with the increase of training time.

Research on the variation diagram of the influence of stimulation frequency on the muscle contraction and the sum of muscle contraction, we can see that energy dynamic changes with the time. In the latter part of the anaerobic training, energy consumption reduces gradually. Anaerobic physiological reaction process gradually increases.

\section{Anaerobic training and survey model of vertical jump and straight line sprint}

In the anaerobic training process, the changes of IAT and $\mathrm{T} / \mathrm{C}$ need to be analyzed using mathematical statistics theory. In statistical analysis, we need to use $\mathrm{T}$ test to test the mathematical statistics results for the accuracy of the data. The expression of $\mathrm{T}$ in the formula is:

$$
T=\frac{b_{1}^{1} / a_{1}^{2}}{b_{2}^{2} / a_{2}^{2}} \sim T\left(k_{1}-1, k_{2}-1\right)
$$

In this formula, $\mathrm{b}$ is variance. $\mathrm{k}$ is sample amount. The two-sided confidence domain of T-test is:

$$
\left(0, T_{1-\frac{t}{2}}\right),\left(T_{\frac{t}{2}},+\infty\right)
$$

The statistics of Anaerobic training vertical jump and the straight sprint training is:

$$
t=\frac{\bar{X} c-C_{0}}{b c / \sqrt{n_{c}}}
$$

In this formula, $C_{0}$ is the assumed difference, and the mean of sample difference of anaerobic training vertical jump and a straight line sprint is: 


$$
\bar{X}_{c}=\frac{\sum_{i=1}^{n} C_{i}}{n_{c}}
$$

The variance of sample difference of anaerobic training vertical jump and a straight line sprint is:

$$
B C=\sqrt{\frac{\sum_{i=1}^{n}\left(C_{i}-\bar{X}_{c}\right)^{2}}{n_{c}-1}}
$$

Nine players in the national youth basketball team are willing to accept the test. Characteristics of the test subjects of athletes are as follows (average \pm SD): Age, 17.0 +/-0.0 years; Weight, 66.1 + /-6.0 $\mathrm{kg}$; Height, $179.2+/-2.7 \mathrm{~cm}$; Body fat $(\%), 10.1$ plus or minus $2.5 \%$. Subjects in the national team training received tests in the days before the testing; therefore we can assume that all of the subjects have very good basketball conditions. All the tests are executed as Wingate test. The Kendall rank correlation coefficient analysis method of test is used to do data processing. The test data are based on the average \pm SD to display. When there are significant level relations ( $p$ than 0.05 ), there is no significant relationship $(\mathrm{p}>0.05)$. In the use of Kendall rank correlation coefficient of the test data in the process, for case $n \leq 30$, the Kendall level can be directly used to relevant statistics, and statistical analysis software can be directly obtained according to the table to corresponding companions probability value. In this study, there are nine test subjects, meaning we can directly use this form, and then use SPSS for analysis.

$$
\tau=\frac{2 P}{\frac{1}{2} n(n-1)}-1=\frac{4 P}{n(n-1)}-1
$$

\section{Research on vertical jump and straight-line sprint anaerobic capacity}

This paper does the anaerobic training of 300 athletes and tests the IAT and the T / C in the training process and does the t- test of the statistical results and gets the sample difference variance before and after the training. At last, the IAT changes of basketball vertical jump and straight sprint anaerobic training are shown in table 1.

Table 1. IAT changes of anaerobic training

\begin{tabular}{l|l|l}
\hline Training time & IAT \pm Bc after vertical jump training & IAT \pm Bc after linear sprint training \\
\hline 0 months & $1.65 \pm 0.25$ & $1.64 \pm 0.23$ \\
1 months & $1.64 \pm 0.28$ & $1.63 \pm 0.24$ \\
2 months & $1.62 \pm 0.33$ & $1.61 \pm 0.30$ \\
3 months & $1.60 \pm 0.31$ & $1.59 \pm 0.28$ \\
\hline
\end{tabular}

From Table 1, we can see that the IAT change of straight line sprint is smaller than the vertical jump which proves that the anaerobic capacity of vertical jump is smaller than the straight-line sprint.

From the table 2, we can see that the T/C change of straight line sprint is larger than the vertical jump which proves that the anaerobic capacity of vertical jump is smaller than the straight-line sprint. The vertical leap and straight sprint will be recorded by Kendall rank correlation tables, with statistical analysis software to draw the following results. The fastest sprint lines are F, G, I, and their average power output and vertical leap are also the highest. In addition, A, C, D in straight line sprint is the 
slowest. In addition, their average power output and vertical leap performance are not good. Kendall $(\tau)$ rank correlation analysis shows that there is a significant positive relationship between the CMJ and T1 $(\tau=0.58)$, T2 $(\tau=0.78)$ and between APJT and T2 $(\tau=0.61)$, and a positive correlation $(\mathrm{p}<0.05)$ between APJT and T3 $(\tau=0.61)$. The relation between MP and T1 $(\tau=0.61)$, T2 $(\tau=0.54)$ is moderate, and PP and linear sprint of relevance is not significant. CMJ and PP $(\tau=0.59)$, MP $(\tau=0.76)$ have a significant positive correlation. However, relationship between APJT and PP $(\tau=0.20)$, MP $(\tau=0.28)$ is not significant $(\mathrm{p}>0.05)$. There is no significant correlation between FILD and FIWAnT $(\tau=0.22$, $\mathrm{p}>0.05)$.

Table 2. $\mathrm{T} / \mathrm{C}$ change of anaerobic training

\begin{tabular}{l|l|l}
\hline Training time & T/C after vertical jump training & T/C after linear sprint training \\
\hline 0 months & $35 \pm 7.1$ & $36 \pm 8.2$ \\
1 months & $37 \pm 9.8$ & $39 \pm 12.7$ \\
2 months & $31 \pm 4.6$ & $34 \pm 6.8$ \\
3 months & $28 \pm 0.31$ & $29 \pm 3.5$ \\
\hline
\end{tabular}

\section{Conclusion}

This paper analyzed the anaerobic capacity of vertical jump and straight-line sprint in basketball athletes training process. We can see that anaerobic capacity of straight line sprint is stronger than anaerobic training process which provides a theoretical reference for the basketball player. The fatigue index between WAnT and linear sprint doesn't have any relationship; this may be due to the fact that the two test movements are caused by different rules. WAnT is a separate movement for 30 seconds. But sprint also has the same three phases as WAnT, but there are 2 minutes to rest. Therefore, FIWanT is to measure a single sports fatigue index, and FILD is to test the recovery probability. If we conduct multiple, discontinuous WAnT test in this study, perhaps the two sports fatigue index will be more similar.

\section{References}

[1] Hiroki Okubo, Mont Hubbard, "Identification of basketball parameters for a simulation model", Procedia Engineering, Vol. 2, No. 2, pp. 3281-3286, 2010.

[2] P. Gouthon, B.A. Tonon, E.M. Ouendo, J.M. Falola, "Évolution des paramètres associés à la fonction rénale après un match de basketball en milieu africain subsaharien", Science \& Sports, Vol. 24, No. 6, pp. 293-301, 2010.

[3] E. Fruchart, P. Pâques, E. Mullet, "Decision-making in basketball and handball games: A developmental perspective", Revue Européenne de Psychologie Appliquée/European Review of Applied Psychology, Vol. 60, No.1, pp. 27-34, 2010.

[4] Fazhi Sun, "Comprehensive Evaluation and Research on Teaching Abilities of Basketball to the Normal Colleges Students Specializing in Basketball of Physical Education", JDCTA, Vol. 7, No. 2, pp. $379-385,2013$.

[5] Y. Blache, A. Beguin, K. Monteil, "Effects of various parameters of basketball shoes on vertical jumping performance: A case study", Science \& Sports, Vol. 26, No.1, pp. 48-50, 2011. 